The horizontal displacement of the loaded end is calculated from Eqs. (1) and (4) with $x=0$ when $\phi=0$. Thus

or

$$
P(L-\Delta)=B\left(\frac{d \phi}{d s}\right)_{\phi=0}=B \sqrt{\frac{2 P}{B}}\left(\sin \phi_{0}\right)^{1 / 2}
$$

$$
\frac{L-\Delta}{L}=\frac{\sqrt{2}}{\alpha}\left(\sin \phi_{0}\right)^{1 / 2} .
$$

From Eq. (6) we have $\sin \phi_{0}=2 k^{2}-1$.

Numerical results can be obtained by: (1) selecting values of $k$ corresponding to tabulated values of the modular angle in the elliptic function tables and (2) determining $\theta_{1}$ and $\alpha$ from Eq. (7). After this has been done, $\delta / L$ and $(L-\Delta) / L$ can be calculated from Eqs. (9) and (10) and plotted against $\alpha^{2}=P L^{2} / B$. The results of these calculations are shown in Fig. 1.

CORRECTIONS TO MY PAPER

\title{
ON THE DEFLECTION OF A CANTILEVER BEAM*
}

Quarterly of Applied Mathematics, 2, 168-171 (1944)

BY H. J. BARTEN

This paper is correct up to the equation

The next step

$$
\theta_{L}=\int_{0}^{L} a s \cos \theta d s
$$

$$
\frac{d \theta_{L}}{d L}=a L \cos \theta_{L}
$$

is incorrect since $\theta$ is not only a function of $L$, but is also a function of $s$. This error makes Eqs. (9), (11), and (12) incorrect.

Using the relation

$$
\frac{d \theta}{d s}=a\left(x_{L}-x\right)
$$

and the various steps used in the original paper, we find that

$$
a^{1 / 2} L=F\left(k, \frac{\pi}{2}\right)-F(k, \delta) .
$$

By using $\delta$ as an independent variable we can calculate corresponding values of $k$ and

* Received June 25, 1945. 


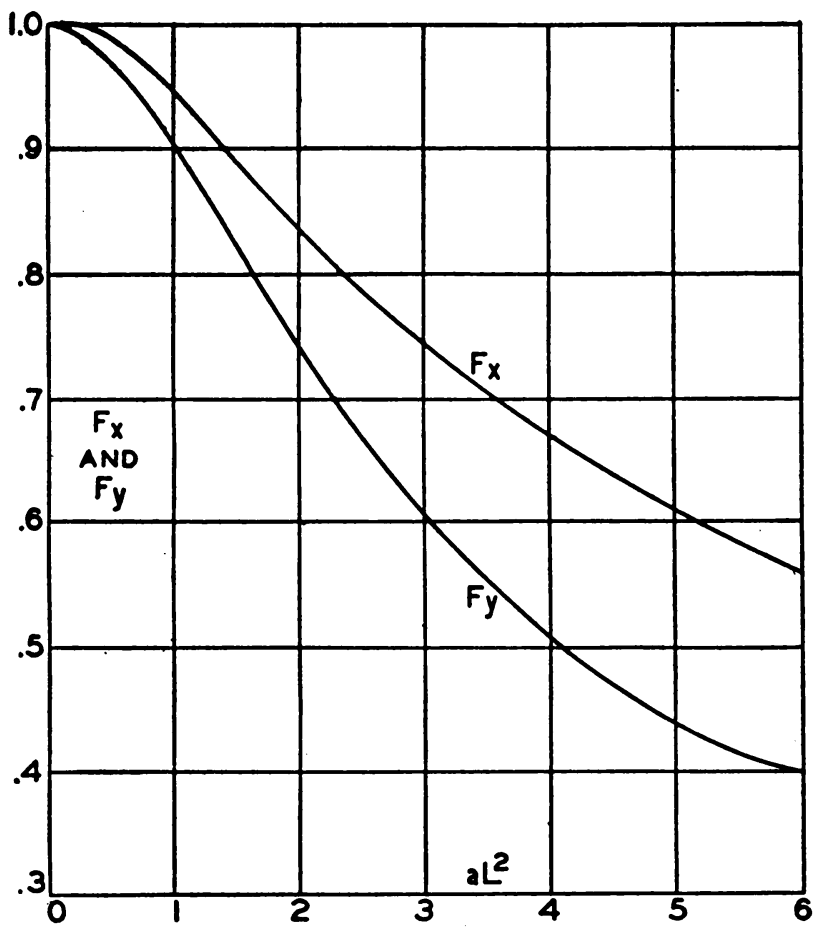

FIG. 1. $a L^{2}$ which in turn are used to find corresponding values of $F_{x}$ and $F_{y}$. The corrected curves thus derived are shown in Fig. 1.

The author wishes to thank M. M. Johnson of Washington, D. C. and D. C. Drucker of the Armour Research Foundation for pointing out these discrepancies.

\section{BIBLIOGRAPHICAL LIST}

The R.T.P. translations listed below are now available from the Durand Reprinting Committee, in care of California Institute of Technology, Pasadena 4, California.

R.T.P. Translations No. 2403, Discontinuous solutions of the equations of motion of fluid flow. By M. Kolscher. 15 pages.

R.T.P. Translation No. 2404, The aero-engine cylinder. By Captain P. Mazzuchelli. 6 pages.

R.T.P. Translation No. 2406, Apparatus for measuring flow turbulence. By G. Daetwyler. 7 pages.

R.T.P. Translation No. 2410, Flight tests with the Poincaré-Farman torque meter. By Jacques Salez. 18 pages.

R.T.P. Translation No. 2411, Blind flying device. By L. Fusier. 6 pages.

R.T.P. Translation No. 2418, Mechanism of the oxidation of hydrocarbons. By M. Prettre. 7 pages.

R.T.P. Translation No. 2419, Theories of spontaneous oxidation. By A. Bach. 7 pages.

R.T.P. Translation No. 2420, Influence of longitudinal high-frequency (supersonic) vibrations on the magnetic behaviour of nickel. By Gerhard Schmid and Ulrich Jetter. 12 pages.

R.T.P. Translation No. 2421, Polyvinylacetal Resins. By H. Gibello. 5 pages. 